\title{
Structural associations between organelle membranes in nectary parenchyma cells
}

\author{
Silvia Rodrigues Machado ${ }^{1}$ - Elisa A. Gregório² Tatiane M. Rodrigues $^{1}$
}

Received: 4 October 2017 / Accepted: 7 January 2018 / Published online: 17 January 2018

(c) Springer-Verlag GmbH Germany, part of Springer Nature 2018

\begin{abstract}
Main conclusion The close association between membranes and organelles, and the intense chloroplast remodeling in parenchyma cells of extrafloral nectaries occurred only at the secretion time and suggest a relationship with the nectar secretion.
\end{abstract}

Associations between membranes and organelles have been well documented in different tissues and cells of plants, but poorly explored in secretory cells. Here, we described the close physical juxtaposition between membranes and organelles, mainly with chloroplasts, in parenchyma cells of Citharexylum myrianthum (Verbenaeceae) extrafloral nectaries under transmission electron microscopy, using conventional and microwave fixation. At the time of nectar secretion, nectary parenchyma cells exhibit a multitude of different organelle and membrane associations as mitochondria-mitochondria, mitochondria-endoplasmic reticulum, mitochondria-chloroplast, chloroplast-nuclear envelope, mitochondria-nuclear envelope, chloroplast-plasmalemma, chloroplast-chloroplast, chloroplast-tonoplast, chloroplast-peroxisome, and mitochondria-peroxisome. These associations were visualized as amorphous electron-dense material, a network of dense fibrillar material and/or dense bridges. Chloroplasts exhibited protrusions variable in shape and extension, which bring them closer to each other and to plasmalemma, tonoplast, and nuclear envelope. Parenchyma cells in the pre- and post-secretory stages did not exhibit any association or juxtaposition of membranes and organelles, and chloroplast protrusions were absent. Chloroplasts had peripheral reticulum that was more developed in the secretory stage. We propose that such subcellular phenomena during the time of nectar secretion optimize the movement of signaling molecules and the exchange of metabolites. Our results open new avenues on the potential mechanisms of organelle contact in parenchyma nectary cells, and reveal new attributes of the secretory cells on the subcellular level.

Keywords Chloroplast remodeling $\cdot$ Membrane association $\cdot$ Nectary $\cdot$ Organelle juxtaposition $\cdot$ Plastidial protrusions

$\begin{array}{ll}\text { Abbreviations } \\ \text { EFN } & \text { Extrafloral nectaries } \\ \text { ER } & \text { Endoplasmic reticulum } \\ \text { RER } & \text { Rough endoplasmic reticulum } \\ \text { PR } & \text { Peripheral reticulum } \\ \text { CPs } & \text { Chloroplast protrusions }\end{array}$

Silvia Rodrigues Machado smachado@ibb.unesp.br

1 Department of Botany, Institute of Biosciences of Botucatu (IBB), São Paulo State University (UNESP), Botucatu, SP, Brazil

2 Center of Electron Microscopy (CME), Institute of Biosciences of Botucatu (IBB), São Paulo State University (UNESP), Botucatu, SP, Brazil

\section{Introduction}

This paper deals primarily with the description of juxtapositions and close proximity of different organelles, mainly chloroplasts, and membrane associations in parenchyma cells of extrafloral nectaries (EFN) in a Verbenaceae species.

Associations between organelles and membrane continuities have been documented in several organisms in the last decades, but their distribution and abundance has not been systematically reported (Wu et al. 2017). Appositions between endoplasmic reticulum (ER) and plasmalemma, more extensively studied, have important functions in cell physiology, including lipid homeostasis, regulation of $\mathrm{Ca}^{2+}$ dynamics, control of organelle biogenesis, and dynamics (Wu et al. 2017), besides regulating cell-signaling pathways and cell development (Gallo et al. 2016). In plants, 
clear and unambiguous associations between ER and plastid envelope (Crotty and Ledbetter 1973; Cran and Dyer 1973; Benayoun and Fahn 1979; Cheniclet and Carde 1985; McLean et al. 1988; Whatley et al. 1991; Holzinger et al. 2007a), ER and mitochondrial outer membrane (Franke and Kartenbeck 1971; Morré et al. 1971), ER and plasmalemma (Cran and Dyer 1973; Robards and Stark 1988), ER and peroxisomes (Cran and Dyer 1973), mitochondria and plastid (Maier and Maier 1968; Montes and Bradbeer 1976; Miyake et al. 1985; Holzinger et al. 2007a, b; Szczepanik and Sowinski 2014), and plasmalemma and nuclear envelope (Carothers 1972) were documented in different tissues and cells. Regarding secretory structures, juxtaposition of ER with the outer membrane envelope of secretory plastids, named periplastidial reticulum (PR), and continuity of PR and the plasmalemma has been described in glands producing oils and resin (Fahn 1979; Bosabalidis and Tsekos 1982; Cheniclet and Carde 1985; Rodrigues et al. 2011; Guo et al. 2013; Possobom and Machado 2017). Investigations on membrane connections and interactions between organelles provide explanation of how transfer and exchange between cell components could be facilitated (Lee et al. 2012; Mehrshahi et al. 2013).

The dynamic nature of chloroplasts under natural conditions is well known. The conversion of chloroplasts to different plastid types (Evert 2006), formation of chloroplast peripheral reticulum (PR) (Szczepanik and Sowinski 2014), and of envelope membrane extensions into the cytosol (Kwok and Hanson 2004; Holzinger et al. 2007a, b; Buchner et al. 2015; Hanson and Sattarzadeh 2011, 2013; Breuers et al. 2012; Schattat et al. 2011; Moser et al. 2015) have been reported in different plant species. However, to the best of our knowledge, studies on chloroplast changes in extrafloral nectaries, at electron microscopy level, are lacking. Studies with this focus could elucidate how plastids coordinate and communicate with other organelles during the process of nectar secretion.

In the course of a study on extrafloral nectaries (EFN) in Citharexylum myrianthum (Machado et al., unpublished), an arboreal species of Verbenaceae, we repeatedly found situations indicative of the close association of membranes and organelles, and of the formation of chloroplast protrusions in parenchyma cells. Therefore, we argued whether these subcellular features were linked to a particular secretory stage.

The purpose of this paper is to describe the nature and extent of membrane juxtapositions and plastid remodeling in the EFN of C. myrianthum, and to explore the putative link between these subcellular features and nectar secretion.

\section{Materials and methods}

\section{Plant material}

Citharexylum myrianthum Cham. has EFN located in the abaxial face of the leaf blade and also in the leaf blade basis, near its junction to the petiole. We collected leaves $(n=5)$ from adult plants $(n=10)$ living in a remnant area of seasonal semideciduous forest located in the Edgárdia farm, in Botucatu municipality $\left(22^{\circ} 52^{\prime} \mathrm{S}, 48^{\circ} 26^{\prime} \mathrm{W}\right)$, central-western region of São Paulo state, southeastern Brazil.

We collected EFN located next to the petiole of fully expanded and sunlit leaves. This study was carried out using EFN at three secretory stages: (a) pre-secretory stage (intumesced EFN without visible secretion on their surface); (b) active in secretion (EFN exhibiting nectar drops on their surface); and (c) post-secretory stage (EFN withered without secretion on their surface). Samples were cut with a razor blade into pieces (about $2 \times 2 \mathrm{~mm}$ ) and were processed for TEM analysis using conventional chemical and microwave fixation techniques.

\section{Conventional chemical fixation}

Samples $\left(2 \mathrm{~mm}^{2}\right)$ were fixed with $2.5 \%$ glutaraldehyde (0.1 M phosphate buffer, $\mathrm{pH} 7.3)$ and left overnight at $4{ }^{\circ} \mathrm{C}$. Then, samples were post-fixed in $1 \%$ osmium tetroxide in the same buffer for $2 \mathrm{~h}$ at room temperature. Samples were dehydrated in a graded acetone series, each for $15 \mathrm{~min}$, embedded in Araldite ${ }^{\circledR}$ epoxy resin under moderate vacuum, and polymerized for $12 \mathrm{~h}$ at $60{ }^{\circ} \mathrm{C}$. Ultrathin sections were stained with uranyl acetate and lead citrate (Reynolds 1963), and examined with Tecnai Spirit transmission electron microscope (FEI Company, Germany) at $60 \mathrm{kV}$.

\section{Fixation by microwave heating}

Pieces of fresh material $\left(2 \mathrm{~mm}^{2}\right)$ were placed in Eppendorf tubes (Sigma T 6524) filled with $2.5 \%$ glutaraldehyde $(0.1 \mathrm{M}$ phosphate buffer $\mathrm{pH}$ 7.3) and disposed in a polystyrene support in the midline of the chamber. A domestic microwave was previously calibrated according to Login et al. (1986). The optimum energy level and duration of exposure were determined by numerous trials, and $700 \mathrm{~W}$ output for $10 \mathrm{~s}$ under $40{ }^{\circ} \mathrm{C}$ was found suitable. Samples were removed immediately after irradiation and placed in a cold buffer solution (Login et al. 1986). Then, the samples were postfixed for $2 \mathrm{~h}$ in $1 \%$ osmium tetroxide $(0.1 \mathrm{M}$ phosphate buffer $\mathrm{pH}$ 7.3) for $2 \mathrm{~h}$ at room temperature, and processed conventionally as described above. 


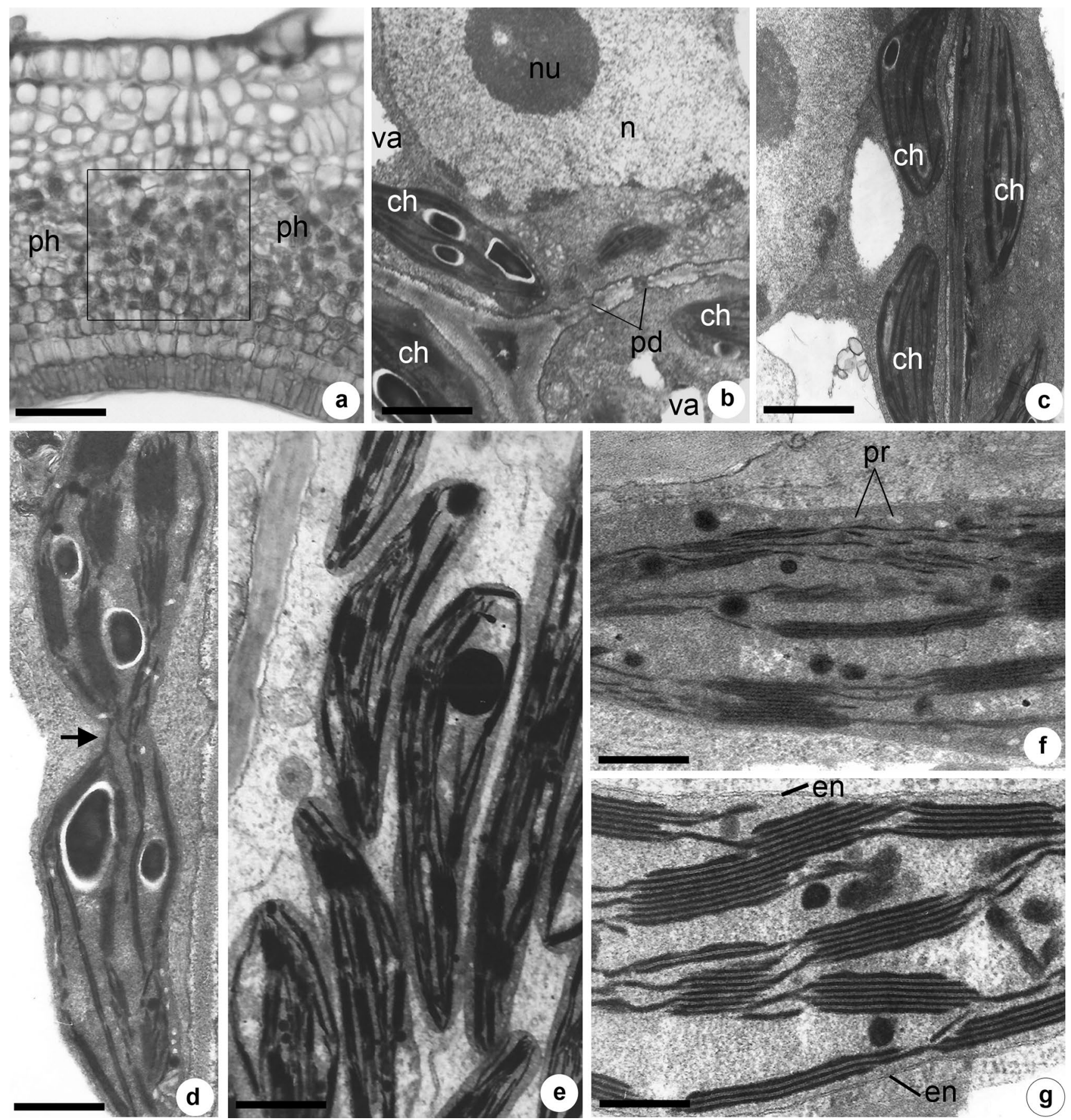

Fig. 1 Extrafloral nectary (EFN) of Citharexylum myrianthum in the pre-secretory stage under light (a) and transmission electron microscopy (b-g); conventional fixation. a Cross section through the EFN showing parenchyma (in the square) vascularized by phloem. b Parenchyma cells exhibiting thin walls with plasmodesmata, voluminous nucleus with nucleolus, small vacuoles, and chloroplasts with starch grains. c Chloroplasts regularly distributed in the cell cortex. d Chloroplast in division with constricted region (arrow). e Abundant and aligned chloroplasts. f Chloroplast with peripheral reticulum, sparse thylakoids, and plastoglobules. g Chloroplast with sparse thylakoids and plastoglobules showing envelope with regular contour. $c h$ chloroplast, en plastidial envelope, $n$ nucleus, $n u$ nucleolus, $p d$ plasmodesmata, $p h$ phloem, $p r$ peripheral reticulum, $v a$ vacuole. Bars $100 \mu \mathrm{m}$ (a), $0.6 \mu \mathrm{m}(\mathbf{b}), 0.5 \mu \mathrm{m}(\mathbf{c}), 0.3 \mu \mathrm{m}(\mathbf{d}), 0.4 \mu \mathrm{m}(\mathbf{e}), 0.2 \mu \mathrm{m}(\mathbf{f})$, and $0.1(\mathbf{g})$ 

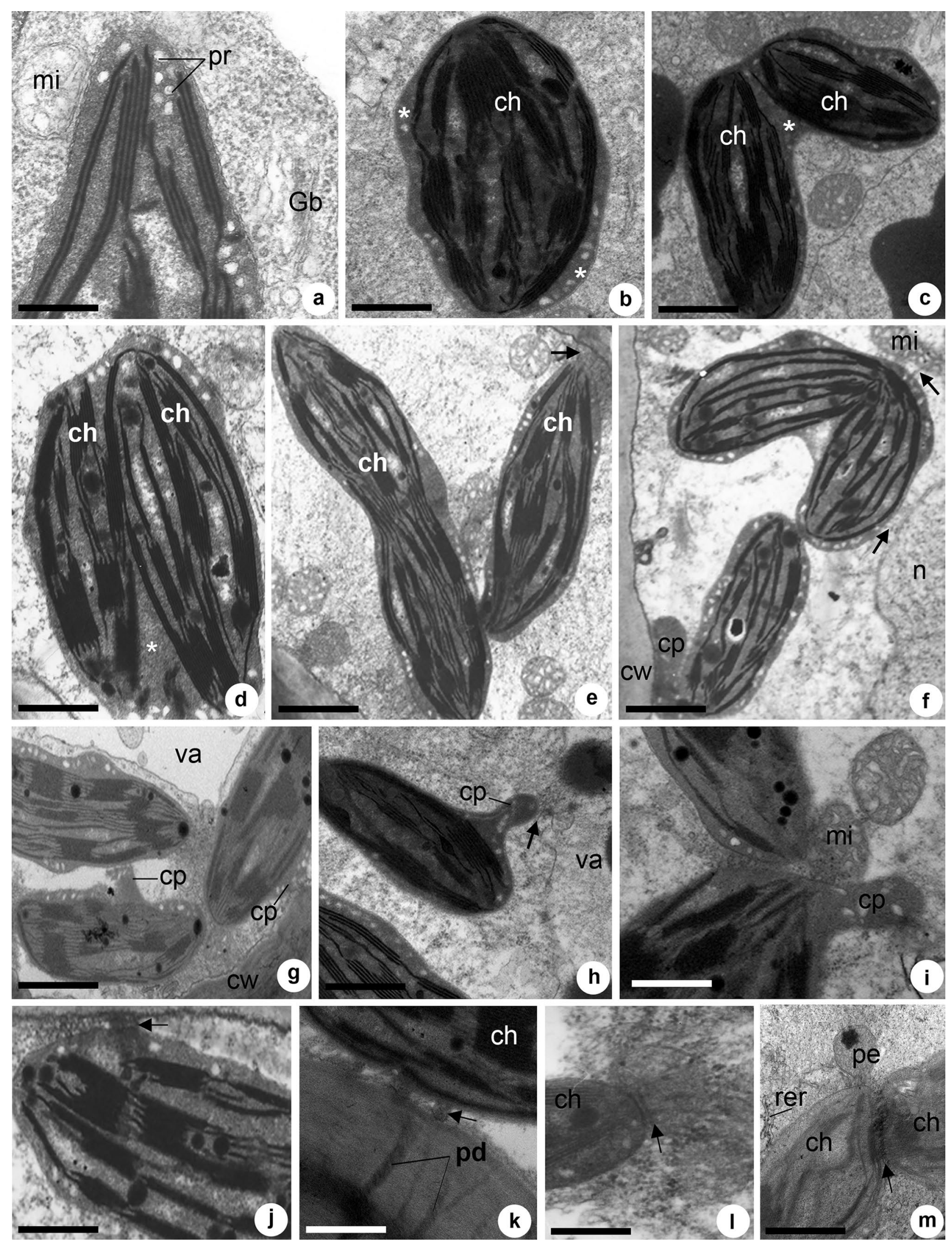
4Fig. 2 Transmission electron micrographs of parenchyma cells from Citharexylum myrianthum EFN at the secretory stage; conventional fixation. a Part of a chloroplast exhibiting developed peripheral reticulum. b Chloroplast irregular in outline due to stromal thickenings (asterisk). c-d Pair of chloroplast laterally joined in the region of stromal thickenings (asterisk). e Association between pair of chloroplasts by their ends. The arrow indicates association chloroplast-plasmalemma by fibrillar material. f Association between chloroplastnuclear envelope, and chloroplast-mitochondria by fibrillar material (arrows). Note chloroplast protrusion closing this organelle to the plasmalemma. g Beak-like chloroplast protrusion bringing near chloroplasts with each other and with plasmalemma. h Chloroplast protrusion close to the tonoplast (arrow). i Chloroplast protrusion close to mitochondria. j Chloroplast-plasmalemma association by dense amorphous material (arrow). k Detail of chloroplast-plasmalemma association by dense material (arrow) near plasmodesmata. 1 Dense network of fibrillar/filamentous material with an intermediary dense plaque (arrow) connecting chloroplasts. m Dense bridges (arrow) connecting chloroplasts. $c h$ chloroplast, $c p$ chloroplast protrusion, $c w$ cell wall, $m i$ mitochondria, $n$ nucleus, $p d$ plasmodesmata, $p e$ peroxisome, $p r$ peripheral reticulum, rer rough endoplasmic reticulum, $v a$ vacuole. Bars $0.15 \mu \mathrm{m}(\mathbf{a}, \mathbf{d}), 0.2 \mu \mathrm{m}(\mathbf{b}, \mathbf{k}), 0.4 \mu \mathrm{m}(\mathbf{c}-\mathbf{h}, \mathbf{l}, \mathbf{m})$, $0.5 \mu \mathrm{m}(\mathbf{g})$, and $0.3 \mu \mathrm{m}(\mathbf{i}, \mathbf{j})$

\section{Results}

The extrafloral nectary of Citharexylum myrianthum was constituted by a secretory uniseriate epidermis with palisade-like cells, a subepidermal region with two layers of hexagonal endodermal-like cells and five-to-six layers of isodiametric and juxtaposed parenchyma cells vascularized with phloem strands (Fig. 1a). We focused our analyses on parenchyma cells, because it was in those cells that we observed changes in membrane juxtapositions and plastid envelope features.

In the pre-secretory stage (Fig. 1), parenchyma cells displayed thin walls with plasmodesmata, voluminous nucleus with evident nucleolus, abundant cytoplasm, and small vacuoles (Fig. 1b). The chloroplasts were ellipsoids, regularly distributed in the cellular cortex, and were featured by undeveloped peripheral reticulum (PR), developed thylakoids and starch grains, some of them with degradation signals (Fig. 1b, c). Chloroplasts with constricted regions suggesting division were commonly observed (Fig. 1d). As the EFN differentiation progressed, the chloroplasts became more elongated, abundant and aligned with each other along their largest axis (Fig. 1e). These newly formed chloroplasts have undeveloped (Fig. 1f) or absent (Fig. 1g) PR, sparse thylakoids, and plastoglobules do not have starch (Fig. 1f, g).

In the secretory stage (Figs. 2, 3), the PR became conspicuous (Fig. 2a). The chloroplasts exhibited localized thickening of the stroma often with PR surrounding the grana, making this organelle's outline irregular (Fig. 2b). Images showing pair of chloroplasts laterally connected along their largest axis (Fig. 2c, d) or by its ends (Fig. 2e, f) were very common in the nectary parenchyma cells.
Some chloroplasts formed protrusions which were commonly filled with stroma and peripheral reticulum (Fig. 2f-i). Chloroplast protrusions, distinct in shape and size, some of them beak-like (Fig. 2g) or wider resembling pseudopods (Fig. 2f, h, i) brought chloroplasts closer to the plasmalemma (Fig. 2f, g), connected chloroplasts between themselves (Fig. 2g), to the tonoplast (Fig. 2h), and to mitochondria (Fig. 2i). Chloroplasts were observed in association with the mitochondria and nuclear envelope by dense filamentous material (Fig. 2f) and with the plasmalemma by dense amorphous material (Fig. 2j, k). Juxtaposition of chloroplast protrusions and plasmalemma occurs mainly near plasmodesmata (Fig. 2k). Associations of chloroplasts to each other also occur by a dense network of fibrillar/filamentous material (Fig. 21), or by dense-like bridge material connecting the outer membranes of the plastid envelope (Figs. 2m, 3a). It was difficult to identify a pattern of organization in the connecting material, but an intermediary electron-dense plaque could be visualized in some chloroplast-chloroplast associations (Fig. 21). Profiles of endoplasmic reticulum are frequently observed near the chloroplast (Fig. $2 \mathrm{~m}$ ) and in close juxtaposition to the plasmalemma (Fig. 3a).

Mitochondria were numerous, large and exhibited welldeveloped cristae (Fig. 3a-h). Association of mitochondria with each other (Fig. 3a, b, d, f), with plasmalemma (Fig. 3b, c, h), with plastids (Fig. 3a, e, h), peroxisomes (Fig. 3f), and with nuclear envelope (Fig. 3g) occurred by dense bridgelike structures.

In the post-secretory stage (Fig. 4), the EFN parenchyma cells presented reduced peripheral cytoplasm and large vacuoles containing dense inclusions and cytoplasmic debris (Fig. 4a). Ellipsoid chloroplasts (Fig. 4a, b), mitochondria with well-developed cristae (Fig. 4c), peroxisome, and rough endoplasmic reticulum (Fig. 4c) were less abundant in relation with the previous secretion stage. At this stage, no juxtaposition of organelles or association between membranes was observed.

\section{Discussion}

Based on TEM studies, this paper describes a multitude of different organelle and membrane juxtapositions, mainly between chloroplasts, in the parenchyma cells of extrafloral nectaries in Citharexylum myrianthum, a Verbenacae species.

Although the occurrence of associations between different organelles and membranes has been widely reported both in animal (Gallo et al. 2016; Wu et al. 2017) and plant (see Mehrshahi et al. 2013 and references cited) cells, this is the first report encompassing a multitude of membrane associations and plastidial remodeling in a secretory structure 

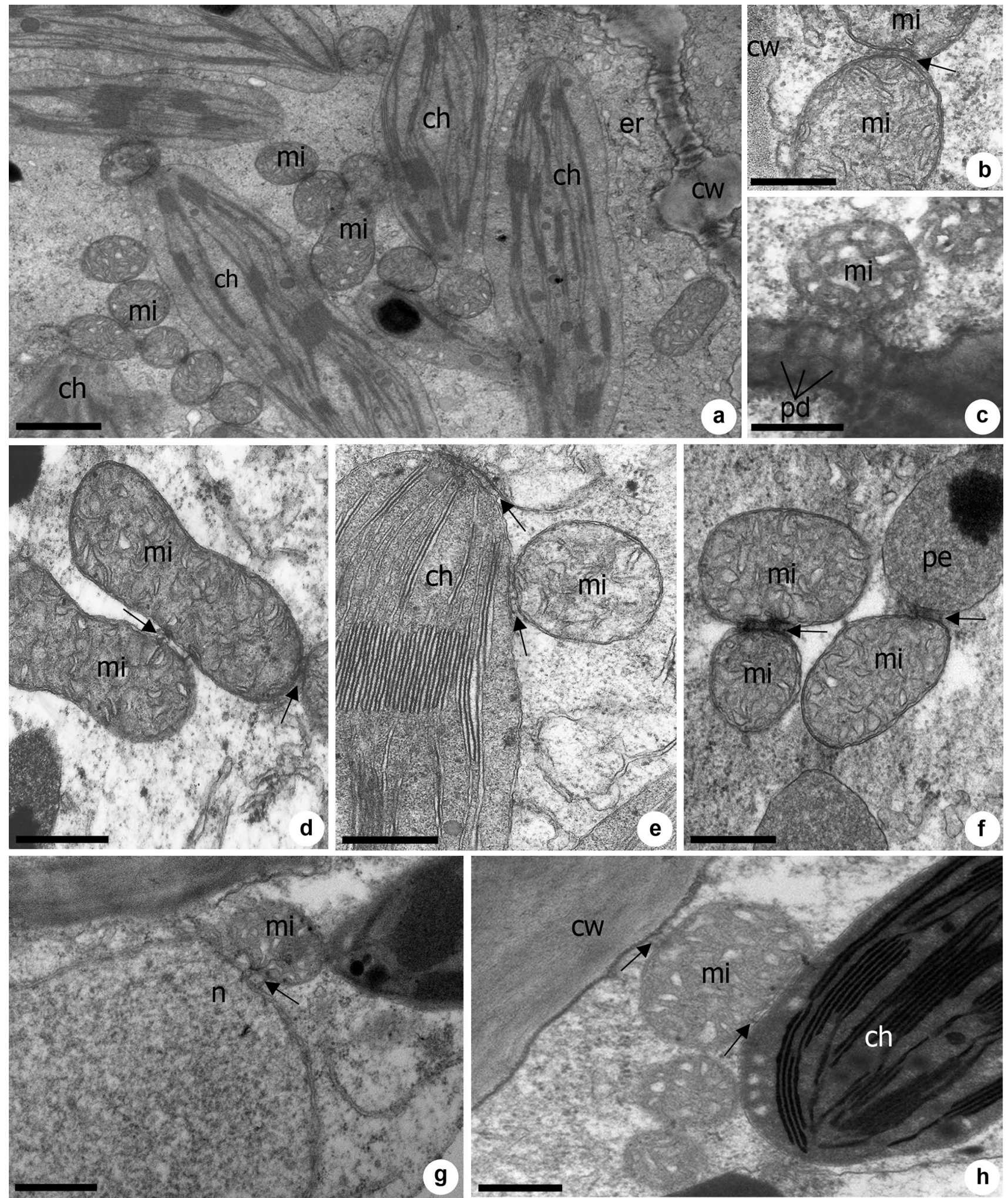

involved in nectar production. As discussed for different systems (Morré et al. 1971; Franke and Kartenbeck 1971; Whatley et al. 1991; Breuers et al. 2012; Mehrshahi et al. 2013; Wu et al. 2017), we proposed that the membrane associations here observed presumably facilitate the interactions and movement of metabolites among organelles linked with nectar secretion. 
4Fig. 3 Transmission electron micrographs of parenchyma cells from Citharexylum myrianthum EFN at the secretory stage. a-f microwave fixation; $\mathbf{g}-\mathbf{h}$ conventional fixation. a Close association among mitochondria and between mitochondria-chloroplast. Note massive amount of endoplasmic reticulum near the chloroplast and the cell wall. b Association between mitochondria by dense bridges (arrow) and mitochondria-plasmalemma. c Association of mitochondria to plasmalemma in the region of plasmodesmata. d Mitochondria associated with each other by dense bridges (arrows).e Mitochondria associated with chloroplast by bridges (arrows). f Mitochondria associated among themselves and with peroxisome by dense bridges (arrows). $\mathbf{g}$ Mitochondria associated with nuclear envelope by bridges (arrow). h Detail of mitochondria in close association with chloroplast and plasmalemma by bridges (arrow). ch chloroplast, $c w$ cell wall, $e r$ endoplasmic reticulum, $m i$ mitochondria, $n$ nucleus, $p d$ plasmodesmata, pe peroxisome. Bars $5 \mu \mathrm{m}(\mathbf{a}), 0.2 \mu \mathrm{m}(\mathbf{b}-\mathbf{h})$ and $0.3 \mu \mathrm{m}$ (g)

When we observed the multitude of membrane associations in EFN of C. myrianthum, we were initially concerned that this could be some artifact induced by the lengthy process of chemical fixation. However, we soon realized that similar structures had been reported sporadically in the literature, and more recently, a clear statement of the existence of close membrane associations, mainly of ER with other intracellular membranes, has been provided (Griffing et al. 2017; Wu et al. 2017). In the present study, close juxtaposition and/or association of membranes were observed in both conventionally fixed samples (immersed in $2.5 \%$ glutaraldehyde fixative for overnight at $4{ }^{\circ} \mathrm{C}$ ) and $\mathrm{MW}$ energy irradiated samples (in conjunction with $2.5 \%$ glutaraldehyde fixative solution) for $10 \mathrm{~s}$ under $40{ }^{\circ} \mathrm{C}$. The combination of MW irradiation and aldehyde allows ultrafast fixation and adequate ultrastructural preservation (Login et al. 1986). Ours results here presented were based on the extensive number of examined samples. By comparing different EFN stages (from pre- to post-secretory stages), we confirmed that the characteristics reported in this paper are genuine and not fixation-induced. In addition, cell wall architecture, nuclear structure, membranes, and cytoplasmic details were well preserved under both fixation methods here employed.

In the EFN parenchyma cells, at the pre-secretory stage, mature chloroplasts have starch grains that decreased in size and abundance toward the secretory stage. Still, in the presecretory stage, after the degradation of the starch grains, chloroplasts become elongated and show signals of division that is identified by increases in chloroplast number and recognition of constricted areas (Robertson et al. 1996). The ultrastructural features of these newly formed chloroplasts suggest that they are not involved with photosynthesis, and are consistent with the multiple functions performed by this organelle. Besides providing nectar carbohydrates through photosynthesis (Nepi 2007), chloroplasts are a center of anabolic metabolism that contain complete biosynthetic pathways (e.g., for de novo synthesis of fatty acids, membrane lipids, amino acids, tocopherols, carotenoids, monoterpenes, and diterpenes) and participate in numerous pathways that span multiple subcellular compartments (Mehrshahi et al. 2013).

We suggest that the chloroplast features here described in the nectary parenchyma cells in the time of nectar secretion, such as absence of starch grains, more developed peripheral reticulum (PR), and formations of chloroplast protrusions (CPs) and stroma thickenings, coupled with the membrane and organelle associations probably occurred in response to physiological demands linked to nectar production. The chloroplast peripheral reticulum (PR), a system of tubules and vesicles continuous with the chloroplast inner membrane (Laetsch 1974), has been reported in leaves of several species (Gracen et al. 1972; Szczepanik and Sowiński 2014), but its occurrence in nectary cells has been underestimated. Some authors have postulated that PR may increase the transport capacity of plastid membranes (Wise 2006), since they increase the surface area of the inner envelope of the chloroplast, which controls the exchange of metabolites between the plastid and the cytoplasm (Heldt and Saur 1971). In this study, chloroplasts have PR, regardless of the EFN secretion stage; however, we found that chloroplasts' PR were more developed in the secretory stage than in pre- and post-secretory stages. This result can reflect the increased demands for metabolite exchange through chloroplast envelopes and cytosol. This interpretation is reinforced by images showing close juxtaposition of chloroplasts with mitochondria and plasmodesmata. The abundance of clustered mitochondria and their juxtaposition with plastids and plasmalemma in the EFN parenchyma cells can be related to energy requirements during starch hydrolysis (Fahn 1979; Nepi 2007) and indicates high metabolic activity rates in the nectary parenchyma. In fact, mitochondria tend to congregate, where energy is required; in cells in which the plasmalemma is very active in transporting substances into or out of the cell, the mitochondria can often be found arrayed along the membrane surface (Taiz and Zeiger 2013). The close apposition of membranes and organelles increasing the overall surface of contact can be associated with the transport of metabolites and informational molecules via symplast (Taiz and Zeiger 2013), besides increases the inter-organelle communication (Gallo et al. 2016). In addition, the abundance of plasmodesmata in the nectary parenchyma cells is related to the pre-nectar transport, via symplast, that is one of the main ways of pre-nectar movement (Nepi 2007).

In this study, the presence of chloroplast protrusions (CPs) connecting plastids with each other, and especially with cellular membranes (plasmalemma, nuclear envelope, and tonoplast) was a remarkable feature in the nectary parenchyma at the time of secretion. Protrusions from the surface of plastids, in distinct sizes and morphological patterns (referred as protuberances, proliferations, chloroplasts protrusions, or stromules) have been observed in a range of 

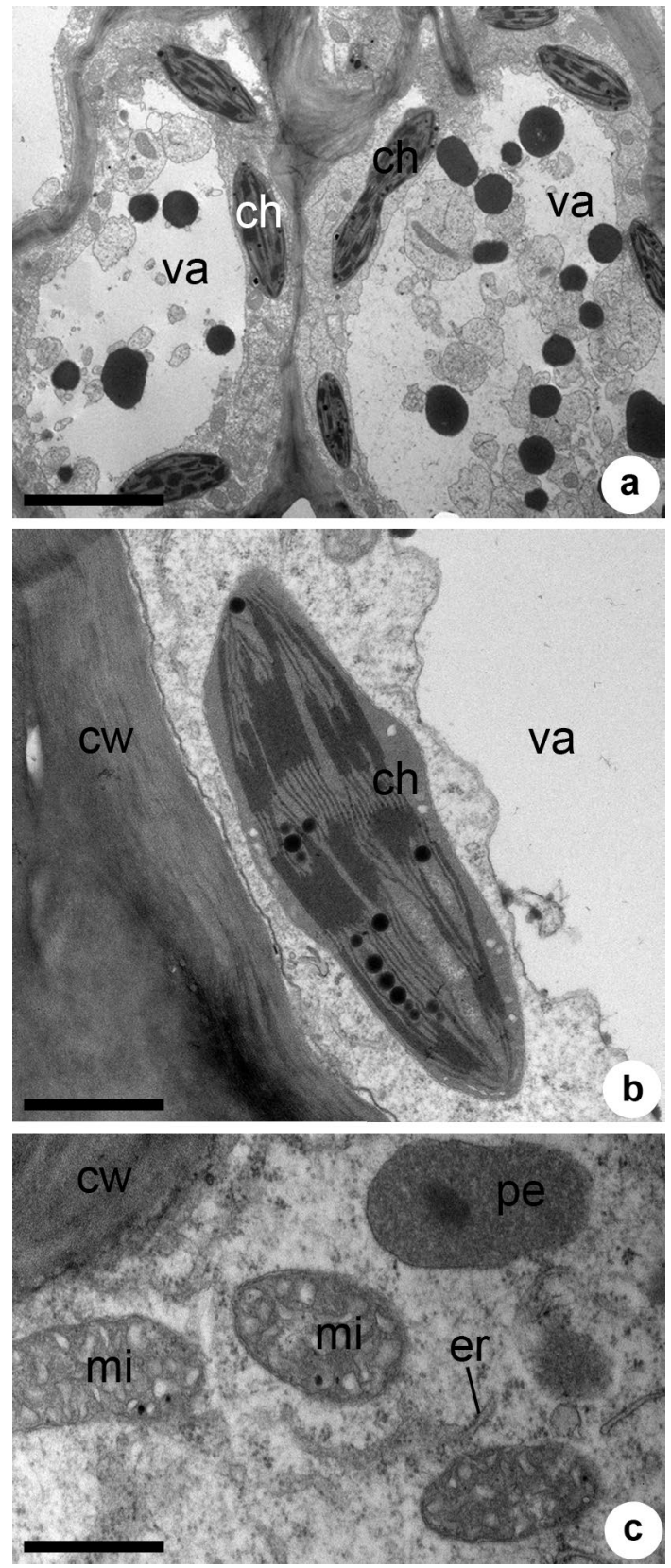

Fig. 4 Transmission electron micrographs of parenchyma cells from Citharexylum myrianthum EFN at the post-secretory stage; conventional fixation. a Overview showing chloroplasts regularly distributed in the reduced peripheral cytoplasm, and large vacuoles containing cytoplasmic debris and dense inclusions. b Chloroplast with thylakoids and plastoglobules in the peripheral cytoplasm. c Mitochondria, peroxisome and endoplasmic reticulum unconnected. $c w$ cell wall, $c h$ chloroplast, er endoplasmic reticulum, $m i$ mitochondria, pe peroxisome, $v a$ vacuole. Bars $2.0 \mu \mathrm{m}(\mathbf{a}), 0.3 \mu \mathrm{m}(\mathbf{b})$, and $0.2 \mu \mathrm{m}(\mathbf{c})$

plant species, plant organs, and plastid types (Gray et al. 2001; Kwok and Hanson 2004; Hanson et al. 2008; Buchner et al. 2015 and references therein). Chloroplast protrusions in close contact with other organelles such as mitochondria and peroxisomes, as here observed, have been interpreted as an adaptation to harsh environments (Lütz and Engel 2007; Holzinger et al. 2007a). These structures are highly dynamic and may be especially sensitive to temperature changes (Holzinger et al. 2007a, b), high salt concentration (Yamane et al. 2012), water stress (Freeman and Duysen 1975), and exogenous application of sucrose or glucose (reviewed in Kumar et al. 2014). Although the formation of CPs and stroma thickenings leads to an enlargement of the chloroplast surface area, their physiological function remains largely unknown, especially regarding the exchange of metabolites and proteins between the plastids (Schattat et al. 2011, 2015; Moser et al. 2015). Our results show that CPs allow chloroplasts to come close to other membranes and organelles in the nectary parenchyma cells, thus increasing the plastid-cytosol contact area. This could facilitate the flow of macromolecules and the transport of chloroplast signals to the nucleus and other subcellular compartments (Caplan et al. 2015; Moser et al. 2015). Therefore, we think that these membrane connections can contribute to rapid transport of metabolites originated of the degradation of starch accumulated in the chloroplasts, which is a physiological demand intimately associated with extrafloral nectar production (Nepi 2007). Our data provide strong evidence that the plastid starch storage is the major source of nectar carbohydrates of extrafloral nectar and that starch grains are hydrolysed to sugar at the secretion stage, as reported for other plant species (e.g., Nepi 2007).

Remarkably, the relationships between chloroplasts are principally external, based on slender electron-dense subcellular plaques and on a dense network of fibrillar or amorphous materials, rather than direct connections between membranes, as in previously published works (e.g., Maier and Maier 1968; Bracker and Grove 1971; Morré et al. 1971; Miyake et al. 1985; McLean et al. 1988; Whatley et al. 1991). Dense bridge-like structures connecting mitochondria with mitochondria, and mitochondria with chloroplasts and peroxisomes, represent an unusual type of membrane connection. Although recent studies indicate the cytoskeletal interaction with membrane compartments as a general phenomenon in plant cells, regulating organelle dynamics and movement, and providing the structural integrity and specificity for various membrane organization/fusion events during plant development (Kwok and Hanson 2003; Wang and Hussey 2015; Griffing et al. 2017), we are not able to interpret the nature of these associations. Recent research with the use of dual and triple fluorescent protein combinations has revealed plastid interactions with mitochondria, the nucleus, the endoplasmic reticulum, and F-actin and suggests integral roles of plastids in retrograde signaling, and cell-to-cell communication (Delfosse et al. 2016). It is important to stress that the results here presented are only indirect indications drawn from chemically fixed materials, and better evidence to elucidate the nature of these 
associations could be obtained by the use of immunofluorescence studies.

Although we have frequently found images of junction of pair of chloroplasts in the parenchyma cells of EFNs in the time of secretion, we are unable to affirm if this is a solely a physical connection or whether there could be some kind of biochemical continuity between the connected plastids, since the concept of interconnected chloroplast has been questioned (Schattat et al. 2015). Remarkably, these interactions were transient and specific to secretory stage of the EFN, since the pre- and post-secretory stages are characterized by unitary, independent plastids. The peculiar cytological features found in the parenchyma cells throughout the EFN secretory cycle (pre, secretory and post-secretion) allow us to make an appreciation of the dynamic nature of the subcellular compartments involved with the nectar secretion process, and highlight the need to pay attention to this region of the nectary in the ultrastructural studies.

In summary, the data on TEM comparative study at the pre, secretory and post-secretory stages of EFN here described indicate that the subcellular peculiarities of the parenchyma cells are temporary and specific to time of nectar secretion. Therefore, we suggest that such associations establish a transient proximity among membranes and thus optimize the inter-organelle trafficking of molecules involved in nectar secretion. However, the underlying mechanisms guiding this process remain unclear. Our results provide interesting information on potential mechanisms for organelle contact in nectary cells, and reveal new attributes of the secretory cells on subcellular level.

Author contribution statement SRM and EAG designed and performed the experiments. All authors analyzed the data, discussed the results, and wrote the manuscript.

Acknowledgements This work was supported by the "Conselho Nacional de Desenvolvimento Científico e Tecnológico'- $\mathrm{CNPq}$ (Grant to S.R. Machado Proc. 02657/2011-8, and Edital MCT/CNPq Proc. 470649/2008-9). We thank the staff of the Electron Microscopy Center of the São Paulo State University (UNESP), Institute of Biosciences, Campus of Botucatu (IBB), for the assistance with sample preparation.

\section{Compliance with ethical standards}

Conflict of interest The authors declare that the research was conducted in the absence of any conflict of interest.

\section{References}

Benayoun J, Fahn A (1979) Intracellular transport and elimination of resin from epithelial duct-cells of Pinus halepensis. Ann Bot 43:179-181
Bosabalidis A, Tsekos I (1982) Ultrastructural studies on the secretory cavities of Citrus deliciosa Ten. II. Development of the oilaccumulating central space of the gland and process of active secretion. Protoplasma 112:63-70

Bracker CE, Grove SN (1971) Continuity between cytoplasmic endomembranes and outer mitochondrial membranes in fungi. Protoplasma 73:15-34

Breuers FKH, Bräutigam A, Geimer S, Welzel U, Stefano G, Renna L, Brandizzi F, Weber APM (2012) Dynamic remodeling of the plastid envelope membranes-a tool for chloroplast envelope in vivo localizations. Front Plant Sci 3(7):10

Buchner O, Moser T, Karadar M, Roach T, Kranner I, Holzinger A (2015) Formation of chloroplast protrusions and catalase activity in alpine Ranunculus glacialis under elevated temperature and different $\mathrm{CO}_{2} / \mathrm{O}_{2}$ ratios. Protoplasma 252:1613-1619

Caplan JL, Kumar AS, Park E, Padmanabhan MS, Hoban K, Modla S, Czymmek K, Dinesh-Kumar SP (2015) Chloroplast stromules function during innate immunity. Dev Cell 34:45-57

Carothers ZB (1972) Studies of spermatogenesis in the hepatical. III. Continuity between plasma membrane and nuclear envelope in andragonial cells of Blasia. J Cell Biol 52:273-282

Cheniclet C, Carde JP (1985) Presence of leucoplasts in secretory cells and of monoterpenes in the essential oil: a correlative study. Isr J Bot 34:219-238

Cran DG, Dyer AF (1973) Membrane continuity and associations in the fern Dryopteris borreri. Protoplasma 76:103-108

Crotty WJ, Ledbetter MC (1973) Membrane continuities involving chloroplasts and other organelles in plant cells. Science $82: 839-841$

Delfosse K, Wozny MR, Jaipargas E-A, Barton KA, Anderson C, Mathur J (2016) Fluorescent protein aided insights on plastids and their extensions: a critical appraisal. Front Plant Sci 6:1253. https://doi.org/10.3389/fpls.2015.01253

Evert RF (2006) Esau's plant anatomy: meristems, cells, and tissues of the plant body-their structure, function, and development, 3rd edn. John Wiley \& Sons, Hoboken

Fahn A (1979) Secretory tissues in plants. Academic Press, London

Franke WW, Kartenbeck J (1971) Outer mitochondrial membrane continuous with endoplasmic reticulum. Protoplasma 73:35-41

Freeman TP, Duysen ME (1975) The effect of imposed water stress on the development and ultrastructure of wheat chloroplasts. Protoplasma 83:131-145

Gallo A, Vannier C, Galli T (2016) Endoplasmic reticulum-plasma membrane associations: structures and functions. Ann Rev Cell Dev Biol 32:279-301

Gracen VE Jr, Hilliard JH, Brown RH, West SH (1972) Peripheral reticulum in chloroplasts of plants differing in $\mathrm{CO}_{2}$ fixation pathways and photorespiration. Planta 107:189-204

Gray JC, Sullivan JA, Hibberd JM, Hanson MR (2001) Stromules: mobile protrusions and interconnections between plastids. Plant Biol 3:223-233

Griffing LR, Lin C, Perico C, White RR, Sparkes I (2017) Plant ER geometry and dynamics: biophysical and cytoskeletal control during growth and biotic response. Protoplasma 254:43-56

Guo J, Yuan Y, Liu Z, Zhu J (2013) Development and structure of internal glands and external glandular trichomes in Pogostemon cablin. PLoS One 8(10):e77862. https://doi.org/10.1371/journal. pone.0077862

Hanson MR, Sattarzadeh A (2008) Dynamic morphology of plastids and stromules in angiosperm plants. Plant Cell Environ 31:646-657

Hanson MR, Sattarzadeh A (2011) Stromules: recent insights into a long neglected feature of plastid morphology and function. Plant Physiol 155:1486-1492

Hanson MR, Sattarzadeh A (2013) Trafficking of proteins through plastid stromules. Plant Cell 25:2774-2782 
Heldt HW, Saur F (1971) The inner membrane of the chloroplast envelope as a site of specific metabolite transport. Biochim Biophys Acta 243:83-91

Holzinger A, Buchner O, Lütz C, Hanson MR (2007a) Temperature sensitive formation of chloroplast protrusions and stromules in mesophyll cells of Arabidopsis thaliana. Protoplasma 230:23-30

Holzinger A, Wasteneys GO, Lütz C (2007b) Investigating cytoskeletal function in chloroplast protrusion formation in the Arctic-Alpine plant Oxyria digyna. Plant Biol 9:400-410

Kumar A, Dinesh-Kumar SP, Caplan J (2014) Stromules. In: Theg S, Wollman F (eds) Adv plant biol: plastid biology, vol 5. Springer, New York, pp 189-207

Kwok EY, Hanson MR (2003) Microfilaments and microtubules control the morphology and movement of non-green plastids and stromules in Nicotiana tabacum. Plant J 35:16-26

Kwok EY, Hanson MR (2004) Stromules and the dynamic nature of plastid morphology. J Microsc 214:124-137

Laetsch WM (1974) The C4 syndrome: a structural analysis. Ann Rev Plant Physiol 25:27-52

Lee HY, Bowen CH, Popescu GV, Kang HG, Kato N, Ma S, DineshKumar S, Snyder M, Popescu SC (2012) Arabidopsis RTNLB1 and RTNLB2 reticulon-like proteins regulate intracellular trafficking and activity of the FLS2 immune receptor. Plant Cell 23:3374-3391

Login GR, Stavinoha WB, Dvorak AM (1986) Ultrafast microwave energy fixation for electron microscopy. J Histochem Cytochem 34:381-387

Lütz C, Engel L (2007) Changes in chloroplast ultrastructure in some high-alpine plants: adaptation to metabolic demands and climate? Protoplasma 231:183-192

Maier K, Maier U (1968) Zur Frage einer Neubildung von mitochondrion aus plastiden. Protoplasma 65:239-242

McLean B, Whatley JM, Juniper BE (1988) Continuity of chloroplast and endoplasmic reticulum membranes in Chara and Equisetum. New Phytol 109:59-63

Mehrshahi P, Stefano G, Andaloro JM, Brandizzi F, Froehlich JE, DellaPenna D (2013) Transorganellar complementation redefines the biochemical continuity of endoplasmic reticulum and chloroplasts. PNAS 110:12126-12131. https://doi.10.1073/pnas.1306331110

Miyake H, Furukawa A, Totsuka T (1985) Structural associations between mitochondria and chloroplasts in the bundle sheath cells of Portulaca oleracea. Ann Bot 55:815-817

Montes G, Bradbeer U (1976) An association of chloroplasts and mitochondria in Zea mays and Hyptis suaveolena. Plant Sci Lett 6:35-41

Morré DJ, Merrit WD, Lembi CA (1971) Connections between mitochondria and endoplasmic reticulum in rat liver and onion stem. Protoplasma 73:43-49
Moser T, Holzinger A, Buchner O (2015) Chloroplast protrusions in leaves of Ranunculus glacialis L. respond significantly to different ambient conditions but are not related to temperature stress. Plant Cell Environ 38:1347-1356

Nepi M (2007) Nectary structure and ultrastructure. In: Nicolson SW, Nepi M, Pacini E (eds) Nectaries and nectar. Springer, Dordrecht, pp 129-166

Possobom CCF, Machado SR (2017) Elaiphores in three Neotropical Malpighiaceae species: a comparative study. Plant Syst Evol. http s://doi.org/10.1007/s00606-017-1443-6

Reynolds ES (1963) The use of lead citrate at high $\mathrm{pH}$ as an electronopaque stain in electron microscopy. J Cell Biol 17:208-212

Robards AW, Stark M (1988) Nectar secretion in Abutilon: a new model. Protoplasma 142:79-91

Robertson EJ, Rutherford SM, Leech RM (1996) Characterization of chloroplast division using the Arabidopsis mutant arc5. Plant Physiol 112:149-159

Rodrigues TM, Teixeira SP, Machado SR (2011) The oleoresin secretory system in seedlings and adult plants of copaíba (Copaifera langsdorffii Desf., Leguminosae-Caesalpinioideae). Flora (Jena) 206:585-594

Schattat M, Barton K, Baudisch B, Klosgen RB, Mathur J (2011) Plastid stromule branching coincides with contiguous endoplasmic reticulum dynamics. Plant Physiol 155:1667-1677

Schattat MH, Barton KA, Mathur J (2015) The myth of interconnected plastids and related phenomena. Protoplasma 252:359-371

Szczepanik J, Sowiński P (2014) The occurrence of chloroplast peripheral reticulum in grasses: a matter of phylogeny or a matter of function? Acta Physiol Plant 36:1133-1142

Taiz L, Zeiger E (2013) Plant physiology, 5th edn. Sinauer Associates Inc. Publishers, Sunderland

Wang P, Hussey PJ (2015) Interactions between plant endomembrane systems and the actin cytoskeleton. Front Plant Sci 6:422

Whatley JM, McLean B, Juniper BE (1991) Continuity of chloroplast and endoplasmic reticulum membranes in Phaseolus vulgaris. New Phytol 117:209-217

Wise RR (2006) The diversity of plastid form and function. In: Wise RR, Hoober JK (eds) The structure and function of plastids. Springer, Dordrecht, pp 3-26

Wu Y, Whiteus C, Shan XuC, Hayworth KJ, Weinberg RJ, Hess HF, De Camilli P (2017) Contacts between the endoplasmic reticulum and other membranes in neurons. PNAS 114:E4859-E4867

Yamane K, Mitsuya S, Taniguchi M, Miyake H (2012) Salt-induced chloroplast protrusion is the process of exclusion of ribulose1,5-bisphosphate carboxylase/oxygenase from chloroplasts into cytoplasm in leaves of rice. Plant Cell Environ 35:1663-1671 